\title{
RISKY MULTI-CRITERIA GROUP DECISION MAKING ON GREEN CAPACITY INVESTMENT PROJECTS BASED ON SUPPLY CHAIN
}

\author{
Yan SONG', Shuang YAO ${ }^{2}$, Donghua YU ${ }^{3}$, Yan SHEN ${ }^{4}$ \\ ${ }^{1,2}$ School of Economics and Management, Harbin Engineering University, \\ No.45 Nantong Street, 150001, Harbin, China \\ ${ }^{3}$ School of Computer Science and Technology, Harbin Institute Technology, \\ No.92 Xidazhi Street, 150001, Harbin, China \\ ${ }^{4}$ College of Science, Harbin Engineering University, No.45 Nantong Street, \\ 150001, Harbin, China \\ E-mails: 1cherry_tigersong@163.com; ${ }^{2}$ alloniam@163.com (correspondingauthor); \\ 33onghuayu@hit.edu.cn; ${ }^{4}$ shenyan@hrbeu.edu.cn
}

Received 02 January 2017; accepted 14 May 2017

\begin{abstract}
Green capacity investment projects have rapidly emerged involving suppliers, customers, and manufacturing organizations in supply chain systems with environmental challenges. This paper focuses on and identifies both primary strategic and operational elements that will aid managers in evaluating and making risky multi-criteria decisions on green capacity investment projects. We propose a cloud prospect value consensus process consisting of feedback and adjustment mechanisms that provide modification instructions to the corresponding decision makers for a decision matrix based on the cloud model and prospect theory, which considers psychological behavior, disagreements between decision makers, and the ambiguity of linguistic variable assessment across multi-criteria risks. The new model increases the efficiency and accuracy of decision making. To verify the feasibility and validity of the Cloud Prospect Value Consensus Degree based on the Feedback adjustment mechanism, its performance is compared with three state-of-the-art multi-criteria group decision-making methods.
\end{abstract}

Keywords: project analysis, decision theory, green capacity investment project, risky multi-criteria, group decision making, prospect theory.

JEL Classification: A12, C44, C60, D81, E22, O22.

\section{Introduction}

As competition is intensifying and markets are becoming global, the exploitation of natural resources is increasing with the rapid process of industrialization. Moreover, frequent business activities are exhausting the limited natural resources and raw materials at a much faster pace than before (Gandhi et al. 2016). Therefore, it is now urgent for industries to develop and implement eco-friendly means to conserve resources and 
achieve sustainable production among all members of the supply chain. It is insufficient to improve the efficiencies of the logistics within an organization; rather, the whole supply chain must be made competitive (Li et al. 2005). Generally, a supply chain is a network of suppliers, manufacturers, distributors, customers, etc. involved in creating a product/service and then integrating the key business processes through the original suppliers that provide products, services, and information with added value for customers and other stakeholders. Capacity investment projects are related to suppliers, manufacturers, and customers. According to the classical SCP (structure-conductperformance) framework, the conduct of enterprises determines their performance. For example, profit-seeking (Jefferson, Xu 1991) behavior can be expected to give rise to improved efficiency, both internal efficiency within the enterprise and allocative efficiency across enterprises. Over longer periods, the allocation of investment enables the most profitable enterprises to expand capacity most rapidly. Even oligopolistic industry firms are confronted with at least two types of ambiguities, including the uncertainty of demand and production technology.

However, environmentally conscious businesses have been receiving increasing scrutiny from both researchers and practitioners. Based on reverse logistics, supply chain theory, and the SCP theory framework, green capacity investment (GCI) projects have three requirements. Firstly, they require effective resource allocation and market clearing. In order to avoid excess capacity and excessive competition, a balance of supply, demand, and capacity equilibrium is required. Secondly, the green supply chain requires the coordination of supply, manufacture, and demand, especially environmental consciousness. Thirdly, to avoid invalid and outdated capacity, the green capacity structure needs to be adjusted according to the requirements of the terminal customers; accordingly, the initial green supplier must be sought to achieve a green and sustainable development chain. In conclusion, GCI projects have special closed relationships and uncertain attributes with multi-states. Traditional enterprise project evaluation tools mainly concentrated on a single side of the supply chain. They did not perform the integrated evaluation and decision making of suppliers, manufacturers, and customers. Therefore, the GCI project selection problem is group decision making of fuzzy multiple attributes. It is essential to analyze and monitor customers and suppliers under a decisive framework to improve the green performance of the whole GCI project supply chain. Meanwhile, group decision making on GCI projects present an opportunity for policymakers and manufacturers to make decisions based on different situations and to place a higher weight on the criteria based on their goals and preferences.

\section{Literature review}

According to the World Bank (2013), despite the new plants, the installed capacity does not currently meet the market demand. Fritsch et al. (2016) pointed out that the lack of an effective regulatory framework for investment, skill shortages, and an inefficient electricity pricing structure continue to be the main constrained factors. Capacity investment has not only been widely used in power projects (Pineda, Morales 2015) but is also closely associated with the environment within the entire sustainable supply chain. 
Vachon and Klassen (2006) examined the impact of environment-related interactions in the supply chain on operational performance. Bowen et al. (2001) proposed a new production modification method aimed at developing and implementing a new pollution preventive technology in order to improve environmental performance. Hussain (2011) presented a modeling framework of different enablers for sustainable supply chains and proposed alternatives for sustainable supply chain development.

Furthermore, some group decision methods have applications to logistics and capacity decisions. Kannan et al. (2009) developed a multi-criteria group decision-making model in a fuzzy environment to guide the process of selecting the best third-party reverse logistics provider. Jackson and Munson (2016) found the key factor that influenced the capacity expansion decision to be the size of the quantity discount offered; however, variability in demand and capacity per unit influenced the expansion decision as well. Group decision making under multi-criteria risk refers to decision problems whose criteria are random variables; that is, the criteria values change with the uncertain environment (Liu, Li 2003; Eskandari et al. 1995). Prospect theory fully considers the psychological factors affecting decision makers (DMs) (Kahneman, Tversky 1979) and effectively corrects their maximum subjective expected utility. This theory has been successfully applied to decision making (Fan et al. 2013; Hansson, Lagerkvist 2014; Meng et al. 2015) and group decision making (Dong et al. 2015; Li, Chen 2014). However, it can only be applied to real numbers and triangular fuzzy numbers, and its applicability to other decision methods (Meng, Chen 2015) is limited. When prospect theory or correlation methods cannot be directly used (Liu et al. 2011), a transformation to linguistic variables is required. The cloud model (Li et al. 2009) transforms the uncertainty relation from the qualitative (linguistic) to the quantitative domain, reflecting both fuzziness (no absolute boundary) and randomness (occurrence probability). The model also integrates the two domains and composes a mapping relation between them. From this perspective, the cloud model is a continuous linguistic variable approach (Ren 2012) that has been used extensively in the field of multi-criteria group decision making (Li et al. 1998; Zhang et al. 2014; Wang et al. 2014). Coordinating these differences requires consistency processing (Li et al. 2009; Wang 2015). TOPSIS (Wang, Lee 2007) is a well-known method for classical MCDM, and some researchers have applied it to solve fuzzy-MCDM problems.

On the linguistic variable group decision problem, DMs participate in decision-making processing. Commonly, three links (or steps) require subjective evaluation, and the participants in each link can be different. First, an initial decision matrix is constructed. This is then weighted according to the preferences of the DMs. If the initial decision matrix does not pass a consistency check, then the DMs reconstruct the initial decision matrix. Two aspects of the existing approach are insufficient: first, group decisions based on linguistic variables are widespread in practice; however, the linguistic variables processed are usually in a single state (Li, Chen 2014), and the probability of the state achieving a multi-state evaluation is unknown. Second, the above research does not combine feedback regulation with the inconsistencies of the DMs. Some approaches have integrated DM feedback (Dong et al. 2015), but if the group evaluation of the DMs 
does not meet the consistency threshold, the DMs perform a reappraisal without any guidance on the correction effect, which is time-consuming and particularly complex for risky multi-criteria group decision making. The number of evaluation iterations can be reduced using automatic negotiation to reach a consensus, but this deviates from the original intention of group decision making. The DMs make only a single appraisal without any feedback, and compromise is realized through the iterative algorithm. This leads to a degree of deviation from the practical decision effect.

In order to do so, this paper proposes a research framework that integrates the cloud model, prospect theory, and risky multi-criteria group decision making on GCI projects. Focusing on the group decision problem of the GCI project with multi-criteria risky linguistic variables, we build models of the cloud prospect consensus degree of multi-criteria risk and feedback regulation. In the next section, the cloud model is used to convert the uncertain linguistic problem to a risky multi-criteria decision problem. In Section 3, the comprehensive prospect decision value is calculated and the cloud prospect decision matrix of all the DMs for all the alternatives is constructed based on prospect theory. The decision matrixes of all the DMs are then aggregated and their consistency measured. Those criteria and alternatives that score less than the acceptable threshold are returned to the DMs along with guidance information to enable correction according to larger divergence attributes. Finally, this method scientifically sorts the alternatives by correcting the decision matrix using feedback from the DMs.

\section{Problem description and theoretical basis}

Consider the group decision problem of multi-criteria risk with the GCI alternatives sets $A=\left\{a_{1}, a_{2}, \cdots, a_{m}\right\}(\mathrm{m} \geq 2)$ and the associated criteria sets $U=\left\{\mathrm{u}_{1}, \mathrm{u}_{2}, \cdots, \mathrm{u}_{n}\right\}(n>2)$, where each criteria is mutually independent. The weight vector of the criteria layer is $\omega=\left\{\omega_{1}, \omega_{2}, \cdots, \omega_{n}\right\}$, which satisfies the constraint condition $\sum_{i=1}^{n} \omega_{i}=1, \omega_{i} \geq 0, i=1,2, \cdots, n$. The possible natural state $f^{j}=\left\{f_{1}^{j}, f_{2}^{j}, \cdots, f_{s}^{j}\right\}$ of criteria $u_{j}$ has probability $p_{l}^{j}$ under $f_{l}^{j}(1 \leq l \leq s)$. For the set of DMs $E=\left\{e_{1}, e_{2}, \cdots, e_{t}\right\}(t \geq 2), x_{i j l}^{k}$ represents the linguistic variable evaluation of alternative $a_{i}$ under criteria $u_{j}$ in the state $f_{l}^{j}$.

\subsection{Cloud model}

The cloud model reflects uncertain phenomena in the field of natural and social sciences. Common forms include normal, triangular, trapezoidal, half, and combined cloud models. Usually, the cloud model (Li et al. 1998) is denoted by a cloud droplet $C(\mathrm{Ex}$, $\mathrm{En}, \mathrm{He})$, where Ex is the expected value, which reflects the mathematical expectation of the qualitative concept, and En is the entropy, reflecting the fuzzy qualitative concept. He is the hyper entropy, which reflects the randomness of dispersion and the degree of certainty. These three numerical characteristics depict the randomness and fuzziness of the qualitative concept.

Definition 1. Suppose that $\mathrm{C}$ is the qualitative concept of a quantitative universe. $\forall x \in U$, the certainty degree $\mu_{C}(x)$ of $C$ is a stochastic number with a stable random tendency. 
Then, the membership degree $\mu_{C}(x)$ of the factor $X$ is called the cloud on the universe $U$, denoted by $C(U)$, namely $\mu_{C}(x): U \rightarrow[0,1], \forall x \in U, x \rightarrow \mu_{C}(x)$.

The normal cloud model is very practical. This paper adopts the normal cloud model, which has the following definition:

Definition 2. Let $\tilde{A}$ be the qualitative concept in the universe $U$. A random instance $x$ in $\tilde{A}, x \in U$, satisfies $x \sim N\left(E x, E n^{\prime 2}\right), E n^{\prime} \sim N\left(E n, H e^{2}\right)$. When the certainty degree of $x$ can be expressed as $y=e^{-\frac{(x-E x)^{2}}{2 E n^{\prime 2}}}$, the distribution of $x$ in the universe $U$ is called the normal cloud.

Definition 3. Let $C_{1}\left(E x_{1}, E n_{1}, H e_{1}\right)$ and $C_{2}\left(E x_{2}, E n_{2}, H e_{2}\right)$ be one-dimensional normal clouds in $U$. The Hamming distance between $C_{1}$ and $C_{2}$ is defined by:

$$
\begin{gathered}
D\left(C_{1}, C_{2}\right)=\sqrt{\frac{\underline{d}^{2}\left(C_{1}, C_{2}\right)+\bar{d}^{2}\left(C_{1}, C_{2}\right)}{2}} . \\
\text { Where } \underline{d}\left(C_{1}, C_{2}\right)=\left|\left(E x_{1}-3 \sqrt{E n_{1}^{2}+H e_{1}^{2}}\right)-\left(E x_{2}-3 \sqrt{E n_{2}^{2}+H e_{2}^{2}}\right)\right|, \\
\bar{d}^{2}\left(C_{1}, C_{2}\right)=\left|\left(E x_{1}+3 \sqrt{E n_{1}^{2}+H e_{1}^{2}}\right)-\left(E x_{2}+3 \sqrt{E n_{2}^{2}+H e_{2}^{2}}\right)\right|
\end{gathered}
$$

In the decision process, the number of clouds has a one-to-one correspondence with the linguistic variable value in $U$. Therefore, most previous studies have used the golden section method to generate clouds ( $\mathrm{Xu}, \mathrm{Li} 2010)$. However, as this approach has some defects, we instead use a method that can reinforce the discrimination.

\subsection{Prospect decision theory based on the cloud model}

The core idea of Prospect Theory is that a prospect decision value, which includes a "value function" and a "weight function", reflects the bounded rationality of the DMs in the decision process. Prospect Theory is considered one of the most important psychological behaviors (Roselló et al. 2014). Based on the cloud prospect decision theory, a cloud prospect decision matrix $V_{i j}^{k}$ for alternative $i$ of attribute $j$ is constructed for every DM. This matrix is composed of a cloud prospect decision value function $v(C)$ and a cloud prospect decision value weight $\pi(p)$. The prospect decision matrix of every DM is

$$
V_{i j}^{k}=\sum_{l=1}^{s} \sum_{z=1, z \neq i}^{m} \pi_{i j l}^{k}\left(p_{l}^{j}\right) v\left(C_{z j l}^{k}\right),
$$

where the value function $v(C)$ is formed by the subjective perception of the DMs:

$$
v\left(C_{i j l}^{k}\right)=\left\{\begin{array}{cc}
\left(D\left(C_{i j l}^{k}, C_{z j l}^{k}\right)\right)^{\alpha}, & C_{i j l}^{k} \geq C_{z j l}^{k} \\
-\lambda\left(D\left(C_{i j l}^{k}, C_{z j l}^{k}\right)\right)^{\gamma}, & C_{i j l}^{k}<C_{z j l}^{k}
\end{array} .\right.
$$

The weight function $\pi(p)$ reflects the probability weight function considering the risk attitude of the DM.

$$
\pi_{i j}^{k}\left(p_{l}^{j}\right)= \begin{cases}\left(p_{l}^{j}\right)^{\tau} /\left[\left(p_{l}^{j}\right)^{\tau}+\left(1-p_{l}^{j}\right)^{\tau}\right]^{1 / \tau}, & C_{i j l}^{k} \geq C_{z j l}^{k}, \\ \left(p_{l}^{j}\right)^{\delta} /\left[\left(p_{l}^{j}\right)^{\delta}+\left(1-p_{l}^{j}\right)^{\delta}\right]^{1 / \delta}, & C_{i j l}^{k}<C_{z j l}^{k}\end{cases}
$$


where $C_{i j l}^{k}$ denotes the cloud evaluation of alternative $i$ of attribute $j$ for every DM. Formula (5) suggests that DM $e_{k}$, for the same attribute $j$ under the same state $l$, compares the size of the cloud droplet for alternatives $i$ and $z(z \neq i)$ and calculates the distance between them $D\left(C_{i j l}^{k}, C_{z j l}^{k}\right)$. As $0 \leq \alpha, \gamma \leq 1$, which represent the degree of concavity and convexity of the regional value function for gains and losses, control the value function $v\left(C_{i j l}^{k}\right)$ of the DM's subjective perception. $\lambda$ is the loss aversion coefficient, when $\lambda>1$, the DM is more sensitive to loss (loss aversion).

The probability weight is according to subjective judgment depending on the possibility $P$ of certain event results. The risk attribute coefficients $\tau$ and $\delta$ control the curvature of the prospect weight function. $\pi(P)$ is the monotone increasing function of probability $P$. When $P$ is very small, the DM could overestimate the slight probability of a given incident; when $P$ is large, $\pi(P)<P$, illustrating that DMs overlook large-probability events (Tversky 1992). In Equation (6), for the same attribute $j$ under the same state $l$, the weight function is controlled by $\tau$, which represents the risk revenue attribute coefficient. The greater this value, the more adventurous the behavior of the DM; in contrast, $\delta$ is the risk loss attribute coefficient.

Equation (4) uses dynamic reference points. Dynamic reference points may contain both "loss" and "income", unlike fixed reference points, which include only one of these factors. An erroneous selection could lead to deviation in the overall results.

\section{Cloud prospect group decision model with feedback adjustment mechanism (CPVCD based on FAM)}

Group decisions are affected by knowledge and preferences, and decisions may have to incorporate serious difference of opinions. On the basis of the cloud model and prospect decision theory, we construct a prospect decision value consistency model that includes the cloud prospect decision value consistency degree and feedback regulation.

\subsection{Cloud prospect value consensus degree (CPVCD)}

CPVCD aggregates the DMs and constructs a prospect decision value consensus degree through two procedures. Essentially, it accomplishes the progressive aggregation of DMs and attribute levels. The aggregation of DMs gives a comprehensive decision result for each alternative $i$ and attribute $j$ for multiple DMs based on the average decision. Based on the cloud model, CPVCD operates at the DM level using a set consistency threshold to provide the preliminary correction scheme.

1) Aggregation. Calculate the cloud prospect decision matrix $V_{i j}^{k}$ of each DM. The prospect decision value matrix of all the DMs for alternative $a_{i}$ is determined by

$$
V_{i j}^{c}=\sum_{k=1}^{t} V_{i j}^{k} / t .
$$

Use the cloud prospect decision matrix to construct the decision matrix of each alternative for each DM, and extract the decision matrix of all DMs according to the alternatives. Finally, use Equation (7) to obtain the cloud prospect group decision matrix $V_{i j}^{c}$ for each alternative $o_{i}$ with attribute. 
2) Prospect Value Consensus Degree based on the cloud model.

As shown in Equation (8), according to the cloud prospect decision matrix $V_{i j}^{k}$. The CPVCD of alternative $i$ for $\mathrm{DM} e_{k}$ is

$$
C P V C D\left(V_{i}^{k}\right)=\sqrt{\frac{1}{n} \sum_{j=1}^{n}\left(\frac{V_{i j}^{k}-V_{i j}^{c}}{\left\{\left[\max _{g}\left(V_{i g}^{k}-V_{i g}^{c}\right)-\min _{g}\left(V_{i g}^{k}-V_{i g}^{c}\right)\right]+\varepsilon\right\}^{*} n}\right)^{2}},(g=1,2, \ldots, n) .
$$

$\varepsilon$ is a correction coefficient, usually assigned a value of 1 . The CPVCD of alternative $i$ for all DMs is computed as:

$$
\operatorname{CPVCD}\left(a_{i}\right)=1-\frac{1}{t} \sum_{k=1}^{t} \operatorname{CPVCD}\left(V_{i}^{k}\right)
$$

Let the consensus degree threshold of CPVCD for group decisions be $\beta$. If $\operatorname{CPVCD}\left(a_{i}\right)<$ $\beta$, then adjust $a_{i}$ according to $\min _{l} C P V C D\left(a_{l}\right), l \leq m$, and select the minimum CPVCD of the alternative to adjust.

\subsection{Feedback adjustment mechanism (FAM)}

The adjustment alternative is determined by the CPVCD model. The feedback adjustment information enables reevaluation at different state levels. To guide the DMs' correction, the greatest divergence of attributes is presented for all the DMs. This feedback adjustment is intended to improve correction efficiency. The operation includes the following four steps.

1. According to the cloud prospect decision matrix $V_{i j}^{k}$, lock the correction scheme $a_{i}$ of CPVCD model, and calculate the average value $V_{i \bar{j}}$ of all the DMs for attribute $j$ based on $V_{i j}^{k}$ :

$$
V_{i \bar{j}}=\sum_{k=1}^{t} V_{i j}^{k} / t \text {. }
$$

2. Calculate the sum of squares of the deviations $\partial_{V_{i j}^{k}}^{2}$ of alternative $i$ and attribute $j$ for
$\mathrm{DM} e_{k}$ : $\mathrm{DM} e_{k}$ :

$$
\partial_{V_{i j}^{k}}^{2}=\sum_{k=1}^{t}\left(V_{i j}^{k}-V_{i \bar{j}}\right)^{2}
$$

3. Inform the DMs of the adjustment to alternative $a_{i}$ that satisfies max $\partial_{V_{i j}^{k}}^{2}$.

4. Calculate the guide information for the feedback adjustment. Compute the cloud prospect decision values according to Equations (1-3). Use the feedback regulation of the preference sequence (case 2) (see Dong et al. 2015 for details). When the prospect decision value $V_{i j}^{k}$ of DM $e_{k}$ for alternative $a_{i}$ with attribute $u_{j}$ is larger than the group decision value $V_{i j}^{c}$, then $\mathrm{DM} e_{k}$ should reduce their original evaluation value. Otherwise, "=" in the formula indicates that the DM could refuse to correct their original evaluation value. This process can be written as follows:

$$
\begin{aligned}
& \text { if } V_{i j}^{k}>V_{i j}^{c} \text {, then }\left(x_{i j l}^{k}\right)^{\prime} \leq x_{i j l}^{k} ; \\
& \text { if } V_{i j}^{k}=V_{i j}^{c} \text {, then }\left(x_{i j l}^{k}\right)^{\prime}=x_{i j l}^{k} ;
\end{aligned}
$$




$$
\text { if } V_{i j}^{k}<V_{i j}^{c} \text {, then }\left(x_{i j l}^{k}\right)^{\prime} \geq x_{i j l}^{k} \text {. }
$$

$\left(x_{i j l}^{k}\right)^{\prime}$ is the linguistic evaluation value after adjustment. Overall, CPVCD and the feedback adjustment can be seen as a complete feedback regulation mechanism. CPVCD determines the consensus degree of the group decision at the alternative level, and determines the program to be modified by comparing the CPVCD of all alternatives. This section subdivides alternatives into attributes and confirms the attribute with the greatest divergence through the maximum deviation. According to the feedback regulation of Equations (12-14), the DMs are directed to reevaluate the alternatives and repeat all the procedures until all alternatives satisfy the consensus degree threshold $\beta$; the DMs can also refuse to reevaluate their decisions. The cloud prospect model is illustrated in Figure 1, and the feedback regulation of the DMs is depicted in Figure 2.

\subsection{Decision procedure}

In summary, under the multi-criteria group decision environment, the group decision procedure based on prospect theory and the cloud model is as follows:

1. The language variables are transformed into a normal cloud model. According to the linguistic evaluation criteria of DMs, the corresponding cloud model is combined with the effective universe $U=\left[X_{\min }, X_{\max }\right]$, as discussed in Section 2.1.

2. A cloud prospect decision matrix is constructed for each alternative. Calculate the cloud prospect decision matrix $\left(V_{i j}^{k}\right)_{m \times n}$ of each DM for each alternative and every attribute according to Equations (1-6).

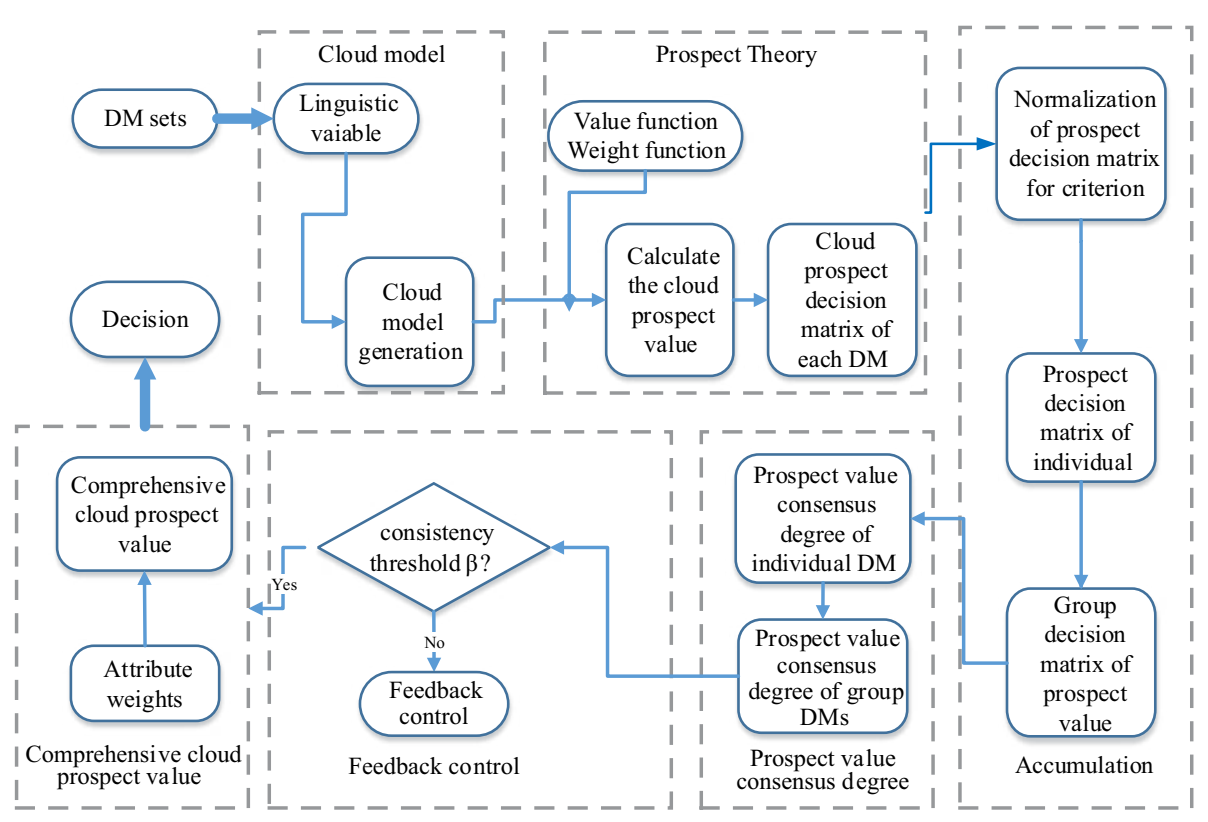

Fig. 1. Group decision model procedure 


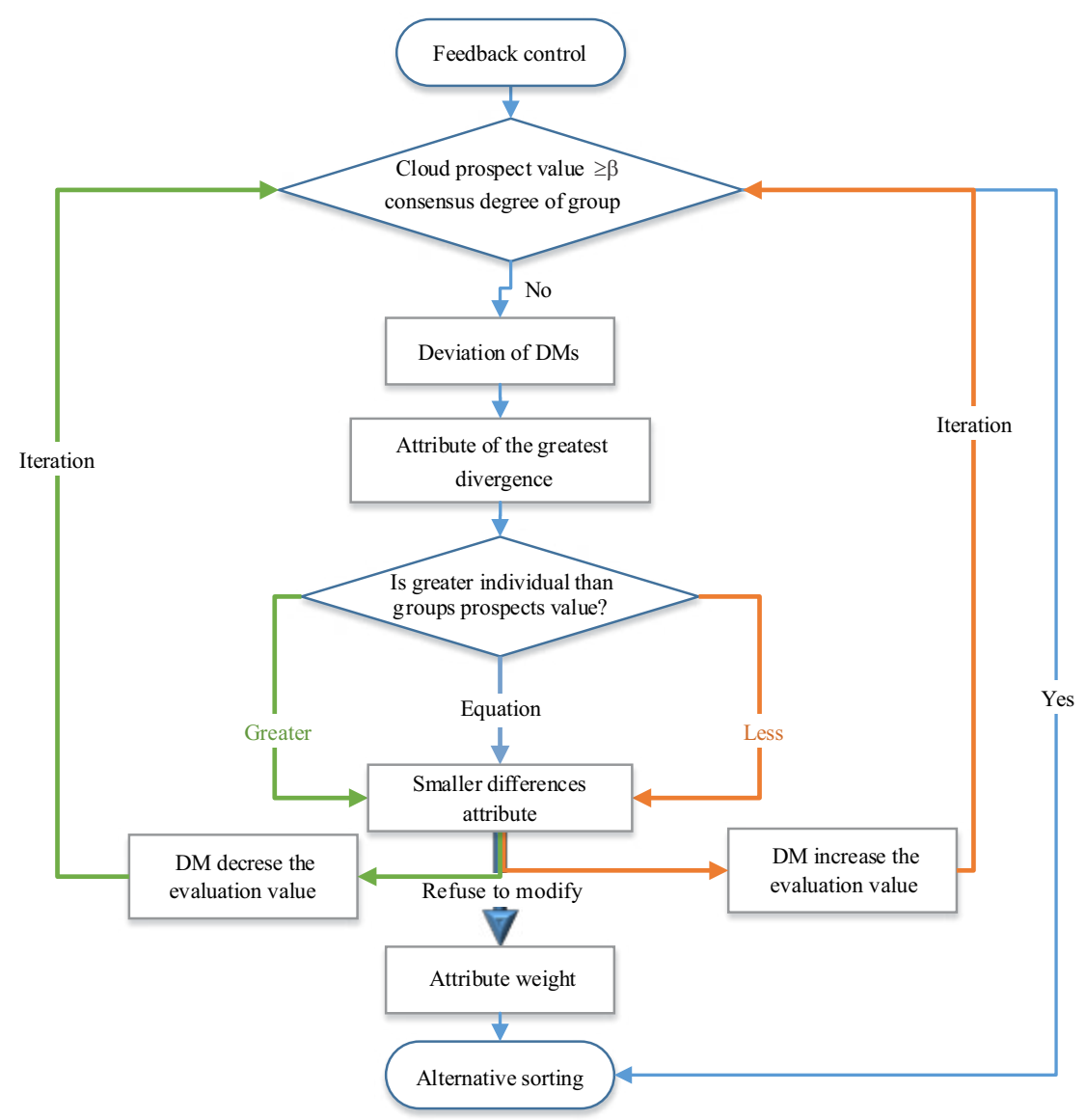

Fig. 2. Feedback control diagram of DMs

3. Aggregate the cloud prospect decision matrixes. Calculate the cloud prospect decision matrix of the group based on the cloud prospect decision matrixes of each DM.

4. Compute the CPVCD. The consensus degree $C P V C D\left(e_{k}\right)$ is calculated for each DM. For a threshold $\beta$, when $C P V C D \geq \beta$ for all alternatives or the DM refuses to correct their evaluation, proceed to step 6; otherwise, proceed to step 5 .

5. Feedback control. Compute the deviation of all the alternatives for different criteria according to Equations (10-11), and provide feedback for the criteria with the greatest deviation according to Equations (12-14). Pass the correction information to the DMs.

6. Compute the comprehensive prospect decision value. Given the weight of attributes $\omega_{j}(j=1,2, \ldots, n)$ for the DMs, we compute the comprehensive prospect decision value of each alternative according to Equation (15), and sort the results based on the comprehensive prospect decision value. The optimal alternative is that with the greatest comprehensive prospect decision value:

$$
V_{i}=\sum_{j=1}^{n} \omega_{j} V_{i j}^{c}, i=1,2, \ldots, m .
$$




\section{Numerical analysis and discussion}

GCI projects in the manufacturing industry are one of the most important international and internal business activities, as they involve large amounts of money, a long period of investment recovery, and a significant degree of risk. Consider an enterprise that intends to invest in one of three production investment projects $o_{i}(i=1,2,3)$. DMs plan to evaluate the four stakeholders of manufacturing enterprises, suppliers, customers, and environment from the perspective of the green supply chain. The detailed criteria are shown in Table 1. These nine criteria are each divided into five possible natural states $f_{l}^{j}(1=1,2, \ldots, 5)$ representing "very good" $\left(f_{1}^{j}\right)$, "good" $\left(f_{2}^{j}\right)$, “fair" $\left(f_{3}^{j}\right)$, "poor" $\left(f_{4}^{j}\right)$, and "very poor" $\left(f_{5}^{j}\right)$. Each attribute has an occurrence probability under the natural state of $f$. The linguistic evaluation set $S=\{1,2,3,4,5\}$ represents \{very poor, poor, fair, good, very good\}. Three experts from marketing, finance, and industry attempt to make a linguistic evaluation. The specific evaluations are listed in Table 1. Finally, according to the cloud prospect decision procedures described above, the optimal GCI project decision is made.

Table 1. Detailed criteria of GCI project decision

\begin{tabular}{lll}
\hline Stakeholders & \multicolumn{1}{c}{ Criteria } & \multicolumn{1}{c}{ Source } \\
\hline \multirow{3}{*}{$\begin{array}{l}\text { manufacturing } \\
\text { enterprises }\end{array}$} & green design and manufacturing & (Li et al. 2015; Gandhi et al. 2016) \\
\cline { 2 - 3 } & reverse logistics & $\begin{array}{l}\text { (Senthil } \text { et al. 2014; Vachon, Klassen 2006; } \\
\text { Kannan } \text { et al. 2009) }\end{array}$ \\
\cline { 2 - 3 } & energy consumption & $\begin{array}{l}\text { (Li } \text { et al. 2015; Fritsch } \text { et al. 2016; Pineda, } \\
\text { Morales 2015) }\end{array}$ \\
\hline \multirow{3}{*}{ suppliers } & green implementation capacity & $\begin{array}{l}\text { (Vachon, Klassen 2006; Geffen, } \\
\text { Rothenberg 2000; Bowen } \text { et al. 2001) }\end{array}$ \\
\cline { 2 - 3 } & quality assurance & (Li et al. 2015; Gandhi et al. 2016; Kuo, \\
& requirements and purchasing power & (Vachon, Klassen 2006) \\
\cline { 2 - 3 } customers & green consumer preference & (Lee et al. 2009; Vachon, Klassen 2006) \\
\hline \multirow{3}{*}{ environment } & environment carrying capacity & (Geffen, Rothenberg 2000; Bowen et al. \\
\cline { 2 - 3 } & financial market state & (Li et al. 2015; Kuo, Lin 2012) \\
\hline
\end{tabular}

Step 1: Transform the linguistic evaluation set to five cloud models on the universe $\mathrm{U}=$ $[-10,10]$, namely $\left\{C_{+2}, C_{+1}, C_{0}, C_{-1}, C_{-2}\right\}$, where the linguistic evaluation set \{very poor, poor, fair, good, very good $\}$ corresponds to $S=\{1,2,3,4,5\}$. The numerical characteristics of the five clouds are outlined in Li et al. (1998), and the corresponding transformation of every attribute is shown in Table 2.

Step 2-Step 3: The cloud prospect decision matrix of individual $V^{k}$ is formed in Step 2, and the cloud prospect decision matrix of group $V^{c}$ is constructed in Step 3. Here, $\tau=$ $0.61, \delta=0.69, \alpha=\gamma=0.88, \lambda=2.25$ (Note: 0.0000 denotes a very small value, not necessarily 0 ; with a higher linguistic evaluation, such as 4 and 5 , there will be negative 
prospect decision value, which does not mean a loss; correspondingly, a positive value does not mean a gain):

$$
\begin{aligned}
V^{1} & =\left(\begin{array}{ccccccccc}
-5.9416 & -5.10263 & -5.50433 & -11.9266 & -0.29873 & -14.9682 & -13.6513 & -7.3887 & -13.6507 \\
-5.64087 & -0.84523 & -12.3644 & 1.958285 & -16.0292 & 2.280272 & 0.480853 & -7.0410 & -9.1677 \\
-11.9936 & -18.9787 & -3.63838 & -5.73521 & -6.06882 & -4.2801 & -7.7734 & -0.4592 & -0.5980
\end{array}\right) ; \\
V^{2} & =\left(\begin{array}{ccccccccc}
-6.3755 & -24.5457 & -0.3984 & 1.1145 & 0.5383 & -2.7534 & -14.8448 & -14.0594 & -14.6422 \\
-6.1072 & 0.2240 & -10.7342 & -4.5837 & -17.8635 & -0.3440 & 2.0450 & -0.1836 & 11.3704 \\
-10.0540 & -3.4638 & -6.7426 & -9.2397 & 1.3666 & -20.2582 & -6.4849 & -4.2245 & -21.3472
\end{array}\right) ; \\
V^{3} & =\left(\begin{array}{ccccccccc}
-14.011 & -0.7117 & -10.7727 & -7.7830 & -1.6968 & -14.2428 & -10.4240 & 8.3509 & 4.4075 \\
-9.8505 & -14.1962 & -11.5341 & -7.2358 & -7.5985 & -15.9996 & -12.7877 & -10.2514 & -11.5960 \\
-7.0658 & -0.3416 & -9.5318 & -22.3345 & -22.4639 & 5.5716 & 3.6125 & -18.1894 & -6.5559
\end{array}\right) ; \\
V^{C} & =\left(\begin{array}{ccc}
-7.1629 & -10.2538 & -2.7046 \\
-5.4695 & -1.1991 & -10.6552 \\
-7.9399 & -9.1961 & -12.0082
\end{array}\right) .
\end{aligned}
$$

Step 4-Step 5: The CPVCD is calculated based on a consensus degree threshold of $\beta=$ 0.9. The individual and group CPVCD are calculated using Equations (8-9). After four

\begin{tabular}{|c|c|c|c|c|c|c|c|c|c|c|c|c|c|c|c|c|c|c|c|c|}
\hline & \multicolumn{5}{|c|}{ U1 } & \multicolumn{5}{|c|}{$\mathrm{U} 2$} & \multicolumn{5}{|c|}{$\ldots$} & \multicolumn{5}{|c|}{ U9 } \\
\hline & $f_{1}^{1}$ & $f_{2}^{1}$ & $f_{3}^{1}$ & $f_{4}^{1}$ & $f_{5}^{1}$ & $f_{1}^{2}$ & $f_{2}^{2}$ & $f_{3}^{2}$ & $f_{4}^{2}$ & $f_{5}^{2}$ & $f_{1}^{3}$ & $f_{2}^{3}$ & $f_{3}^{3}$ & $f_{4}^{3}$ & $f_{5}^{3}$ & $f_{1}^{4}$ & $f_{2}^{4}$ & $f_{3}^{4}$ & $f_{4}^{4}$ & $f_{5}^{4}$ \\
\hline \multicolumn{21}{|c|}{$\mathrm{DM} e_{1}$} \\
\hline$o_{1}$ & 2 & 2 & 2 & 3 & 3 & 2 & 1 & 3 & 2 & 2 & $\ldots$ & $\ldots$ & $\ldots$ & $\ldots$ & $\ldots$ & 5 & 3 & 1 & 2 & 3 \\
\hline$o_{2}$ & 2 & 3 & 1 & 5 & 2 & 1 & 3 & 1 & 4 & 2 & $\ldots$ & $\ldots$ & $\cdots$ & $\ldots$ & $\ldots$ & 4 & 1 & 4 & 3 & 2 \\
\hline$o_{3}$ & 3 & 1 & 3 & 2 & 4 & 2 & 1 & 4 & 2 & 4 & $\ldots$ & $\ldots$ & $\cdots$ & $\ldots$ & $\ldots$ & 2 & 4 & 1 & 2 & 3 \\
\hline$p$ & 0.1 & 0.2 & 0.5 & 0.1 & 0.1 & 0.05 & 0.1 & 0.6 & 0.15 & 0.1 & $\ldots$ & $\ldots$ & $\ldots$ & $\ldots$ & $\ldots$ & 0.3 & 0.15 & 0.1 & 0.25 & 0.2 \\
\hline \multicolumn{21}{|c|}{$\mathrm{DM}_{3}$} \\
\hline$o_{1}$ & 3 & 4 & 2 & 1 & 3 & 4 & 2 & 5 & 2 & 1 & $\ldots$ & $\ldots$ & $\ldots$ & $\ldots$ & $\ldots$ & 4 & 2 & 3 & 4 & 3 \\
\hline$o_{2}$ & 2 & 4 & 1 & 5 & 2 & 1 & 3 & 2 & 1 & 4 & $\cdots$ & $\cdots$ & $\cdots$ & $\ldots$ & $\ldots$ & 3 & 1 & 2 & 3 & 1 \\
\hline$o_{3}$ & 4 & 2 & 1 & 5 & 3 & 2 & 1 & 4 & 2 & 1 & $\ldots$ & $\ldots$ & $\ldots$ & $\ldots$ & $\ldots$ & 5 & 4 & 2 & 2 & 3 \\
\hline$p$ & 0.2 & 0.2 & 0.3 & 0.2 & 0.1 & 0.1 & 0.1 & 0.5 & 0.2 & 0.1 & $\ldots$ & $\ldots$ & $\ldots$ & $\ldots$ & $\ldots$ & 0.3 & 0.25 & 0.1 & 0.2 & 0.15 \\
\hline \multicolumn{21}{|c|}{$\mathrm{DM}_{3}$} \\
\hline$o_{1}$ & 2 & 4 & 3 & 1 & 5 & 2 & 3 & 4 & 1 & 2 & $\ldots$ & $\ldots$ & $\ldots$ & $\ldots$ & $\ldots$ & 2 & 2 & 3 & 3 & 2 \\
\hline$o_{2}$ & 4 & 2 & 1 & 5 & 3 & 2 & 5 & 4 & 1 & 2 & $\ldots$ & $\ldots$ & $\ldots$ & $\ldots$ & $\ldots$ & 3 & 4 & 2 & 3 & 4 \\
\hline$o_{3}$ & 4 & 1 & 3 & 2 & 5 & 2 & 2 & 2 & 1 & 4 & $\ldots$ & $\ldots$ & $\ldots$ & $\ldots$ & $\ldots$ & 2 & 3 & 4 & 3 & 3 \\
\hline$p$ & 0.1 & 0.3 & 0.2 & 0.2 & 0.2 & 0.2 & 0.3 & 0.1 & 0.2 & 0.2 & $\ldots$ & $\ldots$ & $\ldots$ & $\ldots$ & $\ldots$ & 0.2 & 0.2 & 0.2 & 0.25 & 0.15 \\
\hline
\end{tabular}
correction iterations, all the DMs have satisfied the consistent degree threshold. The specific feedback corrections are shown in Figure 3.

Table 2. Linguistic prospect decision matrix 


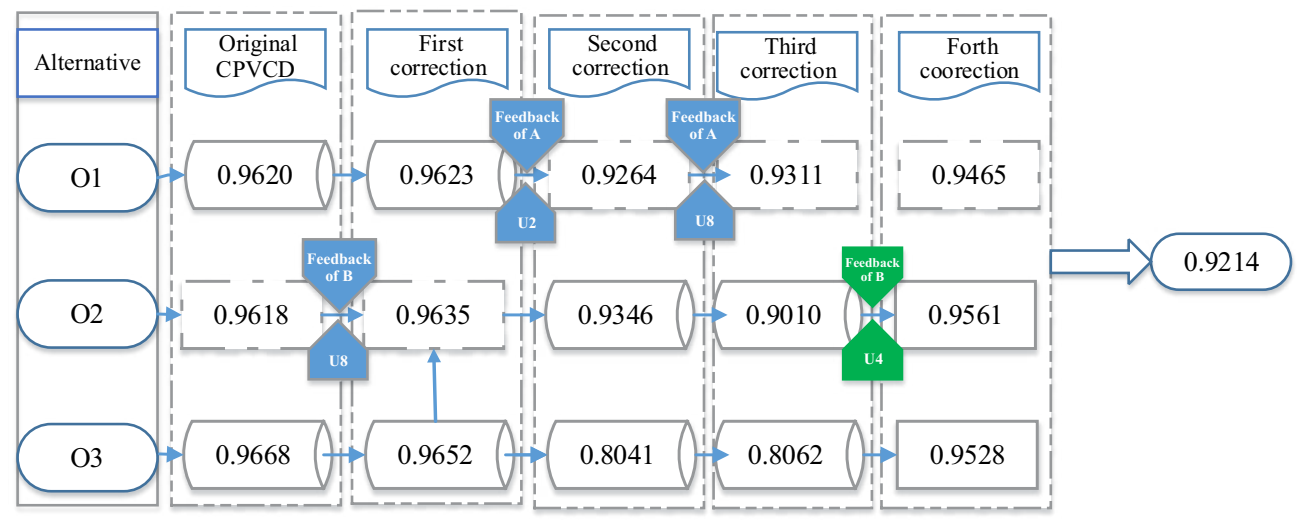

Fig. 3. Feedback correction details

In Figure 3, the cylinders denote that the consistency degree threshold is satisfied and the consistency check is passed. The dashed rectangles represent a consistency degree below the lower limit, which does not pass the check. Although the consistency degree does not meet the threshold in the solid rectangles, this iteration is temporarily permitted. After computing the CPVCD for the group and the three alternatives, although all the three alternatives score more than 0.9 , the group score is 0.8739 . Therefore, according to the feedback adjustment mechanism, the first correction to alternative $o_{2}$ is locked, which is used to define U8 as the reevaluation attribute. The cloud prospect decision value matrix of each DM is calculated according to the feedback adjustment mechanism, and the CPVCDs of the three schemes are obtained after a single correction. At this time, the prospect decision value consistency degree is 0.8976 , an increase of $2.712 \%$, but not high enough to satisfy the consistency threshold. According to the first correction, attribute U2 of alternative $o_{1}$ (which has the minimum CPVCD) is then further locked. By applying the feedback adjustment mechanism again, the second and third corrections cause the consistency of alternatives $o_{1}$ and $o_{2}$ to rise to 0.9633 and 0.9641, respectively. After the fourth correction, the CPVCDs of the three alternatives all have consistency degrees higher than 0.96; what is more, the group consensus degree is higher than the threshold (0.9214). The cloud prospect decision matrixes of the individuals and the group after this correction process are as follows:

$$
\begin{aligned}
& \left(V^{1}\right)^{\prime}=\left(\begin{array}{ccccccccc}
-5.9416 & -17.7469 & -5.50433 & -11.9266 & -0.29873 & -14.9682 & -13.6513 & -7.3887 & -13.6507 \\
-5.64087 & 4.182871 & -12.3644 & 1.958285 & -16.0292 & 2.280272 & 0.480853 & -7.04096 & -9.16769 \\
-11.9936 & -11.7618 & -3.63838 & -5.73521 & -6.06882 & -4.2801 & -7.7734 & -0.45924 & -0.59803
\end{array}\right) ; \\
& \left(V^{2}\right)^{\prime}=\left(\begin{array}{cccccccccc}
-6.3755 & -13.0443 & -0.3984 & 1.1145 & 0.5383 & -2.7534 & -14.8448 & -14.0594 & -14.6422 \\
-6.1072 & -1.9561 & -10.7342 & -4.5837 & -17.8635 & -0.3440 & 2.0450 & -0.1836 & 11.3704 \\
-10.054 & -6.1542 & -6.7426 & -9.2397 & 1.3666 & -20.2582 & -6.6849 & -4.2245 & -21.3472
\end{array}\right) ; \\
& \left(V^{3}\right)^{\prime}=\left(\begin{array}{ccccccccc}
-14.0112 & -16.2169 & -10.7727 & -7.7830 & -1.6968 & -1.6968 & -10.4240 & 12.9802 & 4.4075 \\
-9.8505 & -8.9692 & -11.5341 & -7.2358 & -7.5985 & -15.9996 & -12.7877 & -13.6813 & -11.5960 \\
-7.0658 & 4.2046 & -9.5318 & -22.3345 & -22.4639 & 5.5716 & 3.6125 & -20.8777 & -6.5559
\end{array}\right) ;
\end{aligned}
$$


$\left(V^{c}\right)^{\prime}=\left(\begin{array}{ccc}-9.0721 & -8.5174 & -3.4534 \\ -4.7102 & -1.5283 & -11.0458 \\ -6.8502 & -9.6024 & -12.2465\end{array}\right)$.

Step 6: The attribute weights given by $\mathrm{w}=[0.153,0.151,0.152,0.15,0.05,0.024$, $0.058,0.206,0.057]$ produce a comprehensive prospect decision value of $V=(-9.0721$, $-4.7102,-6.8502)$. Here, the green design and manufacture, reverse logistics, energy consumed, and green complementation are the four most important criteria of the GCI project. According to the maximum principle, the optimal alternative is alternative $o_{2}$. The second best option is $o_{3}$, and the worst comprehensive prospect decision value is $o_{1}$. To verify the proposed cloud prospect consensus degree model, we compared its performance with that of TOPSIS (Wang, Lee 2007), multiple weighted accumulation (MWA) operators (Xu, Li 2010), and the prospect decision value consensus degree (Dong et al. 2015). The results of this comparison are presented in Table 3. With the exception of MWA, the proposed method gives essentially the same decision result as the other methods. In terms of corrections, Wang and Lee (2007) and Dong et al. (2015) mainly adopt the consistency decision and do not employ any correction process. Xu and $\mathrm{Li}$ (2010) construct similarity and accumulation operators for the individual and group decision matrixes. This approach required 37 iterations to satisfy the similarity threshold $\beta$. However, CPVCD based on the FAM needed only four corrections to meet the consensus degree threshold. In terms of the correction mode, TOPSIS and PVCD make the subjective decision once without any correction procedure. Xu's method applies auto-negotiation and does not need any secondary participation in the decisionmaking process. The DMs accomplish the compromise process through an auto-iterative procedure, thus meeting the consistency threshold. This displays a certain deviation from the nature of group decision making. The proposed method uses feedback adjustment, whereby the DMs are presented with specific feedback. This method decreases the number of corrections, cuts down the time costs, and increases the efficiency of decision making. The comparison of the four methods reveals some disparities in the above group decision methods, where the best alternative is similar the same except $\mathrm{Xu}$ and $\mathrm{Li}(2010)$, namely alternative $o_{2}$. However, the worst is between alternatives $o_{1}$ and $o_{3}$, so we choose the ranking order of $o_{2} \succ o_{3} \succ o_{1}$ based on the CPVCD based on the FAM method with the origin of group decision for experts with interpersonal communications and feedback adjustment.

Table 3. Comparison with previous decision-making approaches

\begin{tabular}{lllcl}
\hline \multicolumn{1}{c}{ Literature } & \multicolumn{1}{c}{ Main idea } & Sorting & Correction times & Correction mode \\
\hline Dong et al. 2015 & PVCD & $o_{2} \succ o_{1} \succ o_{3}$ & 0 & None \\
\hline Wang, Lee 2007 & TOPSIS & $o_{2} \succ o_{1} \succ o_{3}$ & 0 & None \\
\hline Xu, Li 2010 & MWA & $o_{3} \succ o_{2} \succ o_{1}$ & 37 & Auto-negotiation \\
\hline CPVCD based-FAM & CPVCD, FAM & $o_{2} \succ o_{3} \succ o_{1}$ & 4 & $\begin{array}{l}\text { Feedback } \\
\text { adjustment }\end{array}$ \\
\hline
\end{tabular}


Further analysis shows the following:

(1) The weight of the attribute differs according to the CPVCD model because of the experts' preference. Although the attributes with higher weights are the same depending on all experts, the attributes with lower weights differ in terms of quality assurance, customer requirements and delivery capacities, green purchase preference, and financial market state.

(2) The DM can refuse to correct. Feedback regulation provides DMs with the ability to reevaluate, essentially instructing the DM to correct their previous evaluation. The DMs could do this to varying degrees according to their own experience. They can also refuse to apply any corrections, although they must give an objective reason for this.

(3) Diverse state of decision making. Linguistic variable evaluation often uses triangular fuzzy numbers or intuitionistic fuzzy numbers (Geffen, Rothenberg 2000; Meng et al. 2015; Li, Chen 2014). However, these methods all conduct linguistic evaluation under a single situation or use multi-attribute risk outside of the group decision process. This paper has not only described how to evaluate multi-situation states containing risky linguistic variables but has also proposed a framework in which prospect theory is used to assign probabilities and weights to the attributes, thus achieving multi-state decisions for the alternatives.

(4) The high efficiency of the decision model. Feedback adjustment not only reduces the number of iterations but also reflects the essence of the group decision, which is the organic integration of subjectivity and objectivity. In terms of a decision result, this method not only approximates the public method but is also highly efficient, requiring fewer iterations to reach the optimal decision.

\section{Conclusions}

The proposed method has elaborated on a new structure to appraise GCI projects according to a cloud prospect group decision model based on the feedback adjustment mechanism, which contains not only customers' green requirements and preferences but also suppliers' green complementation capacity from the perspective of a green supply chain. Through this research, we have affirmed the possibility and the efficiency of integrating the cloud model of fuzzy linguistic evaluation and prospect theory. What is more, to evaluate risky multi-attribute group decisions, we have proposed a framework in which the decision problem is described as a random variable. The decision attribute varies within an uncertain environment which changes with the situation. By formulating the group decision-making process as a fuzzy linguistic variable evaluation, we utilize the advantages of the cloud model and prospect theory to form an organic cloud prospect consistency degree framework. This fusion approach not only ensures the consistency of the group decision but also instructs the DMs to correct the decision matrix through a feedback adjustment mechanism. The applicability and efficiency of the CPVCD based on the FAM were verified through a comparison with other decision results, including correction times and correction modes. Although the research 
enables the implementation of green capacity investment projects and other investment group decision problems, to aggregate the fuzzy linguistic evaluation value, a series of operators and the involvement of the cloud model, prospect theory, and feedback principles have been developed under a variety of situations, since prospect theory mainly involves researching the psychology and behavior of DMs, which is the description of the behavior instead of the rigorous mathematical theory. Currently, granular computing has a strong ability to discriminate information. In the future, we shall explore a more organic fusion of different linguistic evaluations based on prospect theory with multistate after identifying more decision-making information and then modify the feedback adjustment mechanism.

\section{Funding}

This work was supported by the National Natural Science Foundation of China (51409065, 71101034), the project of the Provincial Natural Science Foundation (JJ2016QN0048), and the project of the Provincial Postdoctoral Fund (LBH-Z15047), the project of the Provincial Youth Science Foundation (JJ2016QN0645).

\section{Disclosure statement}

We have no competing financial, professional, or personal interests.

\section{References}

Bowen, F.; Cousins, P.; Lamming, R.; Faruk, A. 2001. The role of supply management capabilities in green supply, Production and Operations Management 10(2): 174-189.

https://doi.org/10.1111/j.1937-5956.2001.tb00077.x

Dong, Y. C; Luo, N; Liang, H. M. 2015. Consensus building in multiperson decision making with heterogeneous preference representation structures: a perspective based on prospect theory, Applied Soft Computing 35: 898-910. https://doi.org/10.1016/j.asoc.2015.03.013

Eskandari, A.; Ffolliott, P.; Szidarovszky, F. 1995. Uncertainty and method choice in discrete multiobjective programming problems, Applied Mathematics and Computation 69(2-3): 335351. https://doi.org/10.1016/0096-3003(94)00145-T

Fan, Z. P.; Zhang, X.; Chen, F. D.; Liu, Y. 2013. Multiple attribute decision making considering aspiration-levels: a method based on prospect theory, Computers \& Industrial Engineering 65(2): 341-350. https://doi.org/10.1016/j.cie.2013.02.013

Fritsch, J.; Poudineh, R.; France, N. 2016. Gas-to-power market and investment incentive for enhancing generation capacity: an analysis of Ghana's electricity sector, Energy Policy 92: 92-101. https://doi.org/10.1016/j.enpol.2016.01.034

Gandhi, S; Mangla, S. K.; Kumar, P.; Kumar, D. 2016. A combined approach using AHP and DEMATEL for evaluating success factors in implementation of green supply chain management in Indian manufacturing industries, International Journal of Logistics 19(6): 537-561. https://doi.org/10.1080/13675567.2016.1164126

Geffen, C.; Rothenberg, S. 2000. Suppliers and environmental innovation: the automotive paint process, International Journal of Operations and Production Management 20: 166-186.

https://doi.org/10.1108/01443570010304242 
Hansson, H.; Lagerkvist, C. J. 2014. Decision making for animal health and welfare: integrating risk-benefit analysis with prospect theory, Risk Analysis 34(6): 1149-1159.

https://doi.org/10.1111/risa.12154

Hussain, M. 2011. Modeling the enablers and alternatives for sustainable supply chain management: Master's thesis. Concordia University Montreal.

Jackson, J. E.; Munson, C. L. 2016. Shared resource capacity expansion decisions for multiple products with quantity discounts, European Journal of Operational Research 253(3): 602-613. https://doi.org/10.1016/j.ejor.2016.03.024

Jefferson, G. H.; Xu, W. 1991. The impact of reform on socialist enterprises in transition: structure, conduct, and performance in Chinese industry, Journal of Comparative Economics 15(1): 45-64. https://doi.org/10.1016/0147-5967(91)90104-2

Kahneman, D.; Tversky, A. 1979. Prospect theory: an analysis of decision under risk, Econometrica 47(2): 263-292. https://doi.org/10.2307/1914185

Kuo, R. J.; Lin, Y. J. 2012. Supplier selection using analytic network process and data envelopment analysis, International Journal of Production Research 50(11): 1-12.

https://doi.org/10.1080/00207543.2011.559487

Kannan, G.; Pokharel, S.; Sasikumar, P. 2009. A hybrid approach using ISM and fuzzy TOPSIS for the selection of reverse logistics provider, Resources, Conservation, and Recycling 54: 28-36. https://doi.org/10.1016/j.resconrec.2009.06.004

Lee, A. H. I.; Kang, H.-Y.; Hsu, C.-F.; Hung, H.-C. 2009. A green supplier selection model for high-tech industry, Expert Systems with Applications 36(4): 7917-7927.

https://doi.org/10.1016/j.eswa.2008.11.052

Li, C. B.; Zhang, J. Y.; Gu, Y. D.; Qi, Z. Q. 2015. Method for fuzzy-stochastic multi-criteria decision-making based on prospect theory and improved TOPSIS with its application, Operations Research and Management Science 2: 92-100.

Li, D. Y.; Liu, C.Y.; Gan, W. Y. 2009. A new cognitive model: cloud model, International Journal of Intelligent Systems 24: 357-375. https://doi.org/10.1002/int.v24:3

Liu, P. D.; Jin, F.; Zhang, X.; Su, Y.; Wang, M. H. 2011. Research on the multi-attribute decisionmaking under risk with interval probability based on prospect theory and the uncertain linguistic variables, Knowledge-Based Systems 24(4): 554-561.

https://doi.org/10.1016/j.knosys.2011.01.010

Li, D.; Cheung, D.; Shi, X.; Ng, V. 1998. Uncertainty reasoning based on cloud models in controllers, Computers Mathematics with Applications 35(3): 99-123.

https://doi.org/10.1016/S0898-1221(97)00282-4

Li, S.; Rao, S. S.; Ragu-Nathan, T.-S.; Ragu-Nathan, B. 2005. Development and validation of a measurement instrument for studying supply chain management practices, Journal of Operations Management 23: 618-641. https://doi.org/10.1016/j.jom.2005.01.002

Liu, P.; Li, W. 2003. Risky multi-objective decision-making theory and its application, Chinese Journal of Management Science 11(6):10-14.

https://doi.org/10.16381/j.cnki.issn1003-207x.2003.06.003

Li, X. H.; Chen, X. H. 2014. Extension of the TOPSIS method based on prospect theory and trapezoidal intuitionistic fuzzy numbers for group decision making, Journal of Systems Science and Systems Engineering 23(2): 231-247. https://doi.org/ 10.1007/s11518-014-5244-y

Meng, F. Y.; Chen, X. H. 2015. An approach to uncertain linguistic multi-attribute group decision making based on interactive index, International Journal of Uncertainty, Fuzziness and Knowledge-Based Systems 23(3): 319-344. https://doi.org/10.1142/s0218488515500130 
Meng, F. Y; Tan, C. Q; Chen, X. H. 2015. An approach to Atanassov's interval-valued intuitionistic fuzzy multi-attribute decision making based on prospect theory, International Journal of Computational Intelligence Systems 8(3): 591-605.

https://doi.org/10.1080/18756891.2015.1036224

Pineda, S.; Morales, J. M. 2015. Capacity expansion of stochastic power generation under twostage electricity markets, Computers \& Operations Research 70: 101-114.

https://doi.org/10.1016/j.cor.2015.12.007

Ren, J. 2012. Linguistic-stochastic multi-criterion decision-making method based on cloud model, Computer Integrated Manufacturing Systems 12: 2792-2797.

https://doi.org/10.13196/j.cims.2012.12.216.renj.013

Roselló, L.; Sánchez, M.; Agell, N.; Prats, F.; Mazaira, F. A. 2014. Using consensus and distances between generalized multi-attribute linguistic assessments for group decision-making, Information Fusion 17: 83-92. https://doi.org/10.1016/j.inffus.2011.09.001

Senthil, S.; Srirangacharyulu, B.; Ramesh, A. 2014. A robust hybrid multi-criteria decision making methodology for contractor evaluation and selection in third-party reverse logistics, Expert Systems with Applications 41(1): 50-58. https://doi.org/10.1016/j.eswa.2013.07.010

Tversky, D. K. 1992. Advances in prospect theory: cumulative representation of uncertainty, Journal of Risk and Uncertainty 5: 297-323. https://doi.org/10.1007/BF00122574

Vachon, S; Klassen, R. D. 2006. Green project partnership in the supply chain: the case of the package printing industry, Journal of Cleaner Production 14(6-7): 661-671.

https://doi.org/10.1016/j.jclepro.2005.07.014

Wang, J. Q.; Peng, L.; Zhang, H. Y.; Chen, X. H. 2014. Method of multi-criteria group decisionmaking based on cloud aggregation operators with linguistic information, Information Sciences 274(1): 177-191. https://doi.org/10.1016/j.ins.2014.02.130

Wang, J. Q.; Peng, J. J.; Zhang, H. Y.; Liu, T.; Chen, X. H. 2015. An uncertain linguistic multicriteria group decision-making method based on a cloud model, Group Decision and Negotiation 24(1):171-192. https://doi.org/10.1007/s10726-014-9385-7

Wang, Y. J.; Lee, H. S. 2007. Generalizing TOPSIS for fuzzy multiple-criteria group decisionmaking, Computers \& Mathematics with Applications 53(11): 1762-1772.

https://doi.org/10.1016/j.camwa.2006.08.037

World Bank. 2013. Energizing Economic Growth in Ghana [online], [cited 29 December 2014]. Available from Internet: http://www-wds.worldbank.org/external/default/WDSContentServer/ WDSP/IB/2013/07/22/000442464_20130722120043/Rendered/PDF/796560WP0P13140Box03 77384B00PUBLIC0.pdf

$\mathrm{Xu}$, Y. J.; Li, D. 2010. Approach to reaching consensus in multiple attribute group decisionmaking, Control and Decision 25(12): 1810 -1820. https://doi.org/1001-0920(2010)12-1810-05

Zhang, L. Y; Li, T.; Xu, X. H. 2014. Consensus model for multiple criteria group decision making under intuitionistic fuzzy environment, Knowledge-Based Systems 57: 127-135.

https://doi.org/10.1016/j.knosys.2013.12.013 
Yan SONG received the B.Sc. and M.S. degree in the college of Computer Science and Technology in 1995 and 1999, respectively, and the $\mathrm{PhD}$ degree in Management Science and Engineering from the Harbin Engineering University, Harbin, China, in 2004. From 2004 to 2008, she was Postdoctoral at School of Management, Harbin Institute of Technology, during 2005 to 2006, she was academic visitor at School of Economics, Tilburg University, Holland. She is the editorial board member of ISCRAM Journal. She also is the founder of international conference of ISCRAM-CHINA and the chairman of Academic committee. She current is interested in decision analysis, data mining, risk management and their applications.

Shuang YAO received the B.Sc. degree in Information Management and System from the College of Computer Science and Technology in Northeast Forestry University, Harbin, China, in 2012. She is currently a successive postgraduate and doctoral candidate in School of Economics and Management, Harbin Engineering University, Harbin, China. Her current research interests data mining, decision making.

Donghua YU received the B.Sc. and M.S. degree in science from the College of Science, Harbin Engineering University, Harbin, China, in 2012 and 2015, respectively. He is currently a PhD candidate in School of Computer Science and Technology, Harbin Institute Technology, Harbin, China. His current research interests include data mining, machine learning, bioinformatics and their related applications.

Yan SHEN received the PhD degree in Control Theory and Control Engineering from College of Automation of Harbin Engineering University, Harbin, China, in 2005. In 1993, she became a Lecturer with the College of Science, Harbin Engineering University, Harbin, China, where she has been a Professor since 2005. Her current research interest include numerical analysis and optimization, information processing and their related applications. 